\title{
Accounting for renewable energy supply intermittency in energy systems modelling
}

\author{
$\underline{\text { Simon Dunstall }}^{\text {a }}$, Tarek ElGindy ${ }^{\text {a }}$, Nahid Jafari ${ }^{\text {a b }}{ }^{\text {, Melanie Ayre }}{ }^{\text {a }}$, Andreas T. Ernst ${ }^{\text {a }}$, \\ Paul Graham ${ }^{c}$, Luke Reedman ${ }^{\mathrm{c}}$, Martin Savelsbergh ${ }^{\mathrm{d}}$ and Stuart Woodman $^{\mathrm{a}}$ \\ ${ }^{a}$ CSIRO Computational Informatics, Private Bag 33, Clayton South, 3169, Australia \\ ${ }^{\mathrm{b}}$ School of Mathematics and Geosciences, RMIT University, Melbourne, Australia \\ ${ }^{\mathrm{C}}$ CSIRO Energy Technology, Newcastle, Australia \\ ${ }^{\mathrm{d}}$ University of Newcastle, Callaghan, Australia \\ Email: simon.dunstall@csiro.au
}

\begin{abstract}
We report on the development and application of an integer programming model for simultaneously proposing electricity transmission system augmentations and the selection of electricity generation projects over multi-year time horizons. Undertaking network design while simultaneously addressing renewable energy supply variability over time and space is a difficult proposition. It was however a key design requirement for this model, due to the model's intended use as part of studies of plausible scenarios for Australia's energy future to 2050. The model has been successfully utilised within a multi-stage modelling workflow, being preceded by an economic model of the energy sector and followed by a high resolution simulation of the national electricity market. Together the three models flesh out the details of electricity systems of the future, in terms of the location and technologies for individual power plants, the magnitude of transmission line capacity expansions, and the energy and financial flows in the resulting networks. We present the results from various analyses that have been undertaken using the model, with emphasis on the integration of renewable and fossil-fuel supply options to meet end-user demand, and also describe some of the mathematical and systems-development work that was required in order to yield a model which was sufficiently detailed, solvable in reasonable time, and able to be embedded in a powerful yet easy-to-use GIS and database system.
\end{abstract}

Keywords: Electricity transmission and generation, integer programming, network design 


\section{INTRODUCTION}

Many models used for electricity system analysis are not formulated for considering large numbers of alternatives for generation sources (location, type, and capacity) and transmission network configurations (transmission lines, connection points, and transmission line capacities). More typically the power system and/or market system is modelled in detail but with relatively few network options in play. Other models (including the CSIRO Energy Sector Model, ESM) tend to be more economically-focused and/or spatially aggregated, this leading to more approximate appraisal of details, costs and energy flows in future networks. This paper describes an integer programming model devised for simultaneously proposing electricity transmission system augmentations and the selection of electricity generation projects over multi-year time horizons, for the Australian East coast interconnected network (the National Energy Market, NEM), at an "intermediate" level of spatial detail. The model is implemented as part of a larger software product that covers network design and analysis for freight transport, ports, agriculture and electricity. The software suite, known as the Infrastructure Futures Analysis Platform (IFAP), integrates a database system based on SQL Server, a Geographic Information System (GIS) and a library of data processing routines and optimisation models.

Options for the representation of electrical power flow in transmission network decision models are described in Romero et al. (2002), and include the DC approximation and transportation models. Full AC models are not practical for network design. The DC approximation is a popular choice in single-timestep models. Overbye et al. (2004) show that it is often a reasonable approximation to AC power flow. For large networks that change over time and involve many design decisions, the need to represent each link flow with two continuous variables (power and phase angle) and a binary variable (to represent link connection to a junction) is computationally onerous. For this and other reasons, whole-of-system futures modelling in Australia tends to be addressed using a transportation model at the decision stage, e.g., refer ROAM Consulting (2011). Our longerterm interest is on utilising DC approximations but at present we use a transportation model because a "DC approximation" version of the model has so far proven to be computationally prohibitive to solve. Furthermore, new transmission system construction might be AC or High Voltage DC (HVDC) in future: AEMO's recent modelling for a entirely renewables-based electricity system (Australian Energy Market Operator, 2013) specifies some new HVDC and some new AC inter-regional transmission.

Binato et al. (2001) present a MIP formulation for transmission network expansion using the DC approximation and explores the use of decomposition techniques to solve it, while in a companion paper a search heuristic is utilised (Binato et al., 2001b). The Regional Energy Deployment System, ReEDS, developed by the US National Renewable Energy Laboratory (Short et al., 2011), has similarities to our model and ESM. ReEDS optimizes the regional expansion of generation and transmission capacity, and considers various generation types in designing a least-cost electricity system. The model addresses electricity transmission by using a pre-determined flow upper bound for electricity transfer between regions. Additional transmission capacity can be purchased for a certain cost per MW-mile. Grid connection costs are also applied in ReEDS.

\section{BASIS OF THE MODEL}

The development of our model has been motivated by the need to study questions about the Australian electricity grid of the future. In this, the future extends to 2050 and we are interested in the "trajectory" of the system over time - in terms of the location, type and capacity of generation assets and their transmission-level interconnections - rather than only the system end-state at 2050. The model is applied to:

- Disaggregate state-level energy generation mixes (i.e., installed capacity by generation technology and year) to a smaller spatial scale (i.e., at the level of the zones used by the Australian Energy Market Operator, AEMO, for planning);

- Address intermittency of renewable supply, through considering system power flows and balances at instants of high "network stress" (i.e., where instantaneous demand approaches the level of available supply);

- Investigate options for interconnector (major transmission line) capacity strengthening, including requirements associated with tapping regions with high renewable energy supply potential;

- Capture information about the evolution of the future network over time, by considering annual capacity decisions in the network;

- Undertake a supply-demand balance at a higher level of detail than what is available in economic models, but which does not aim to replicate the function of market simulation models. 
Our model addresses time in terms of annual timesteps over a multiple-year horizon (typically 2015 to 2051). Capacities are determined for each year (i.e., for each timestep) for both transmission and generation assets. Changes in transmission and generation capacities require one or more timesteps to complete, and capacitychange events notionally occur at the beginning of each timestep. The electricity system is represented by generation facilities, transmission lines, zonal hubs (junctions in the transmission system) and demand centres (one in each zone). There are 16 zones in the network representation. These partition points on the NEM strictly in accordance with AEMO in its National Transmission Network Development Plan (NTNDP) documents (Australian Energy Market Operator, 2012). To facilitate geographic calculations we have, where possible, set boundaries for each zone so they coincide with those of Statistical Sub-Divisions (SSDs). Figure 1 shows the NTNDP zones mapped along with NEM transmission and generation assets.

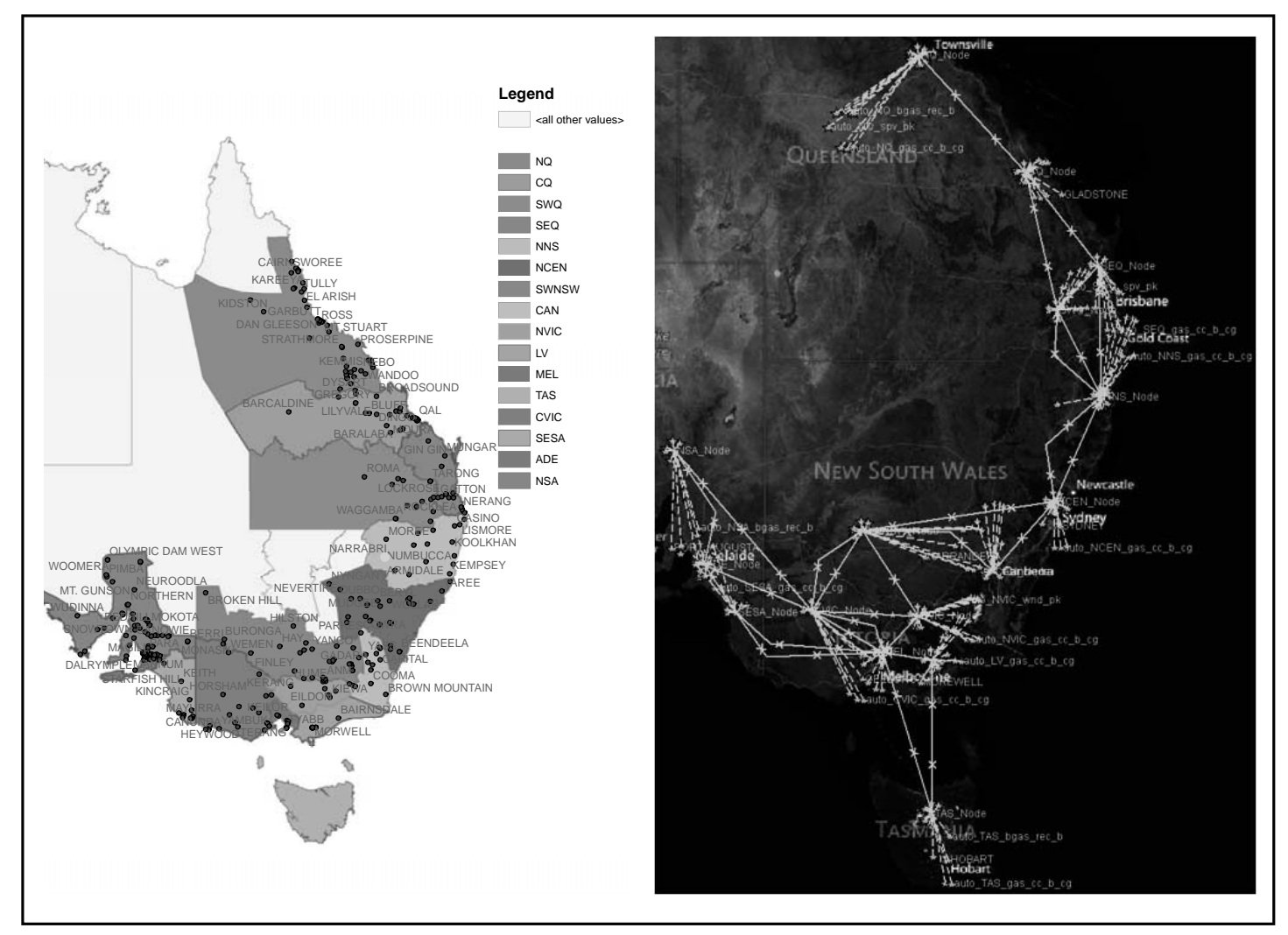

Figure 1. NTNDP zones (left) and NEM transmission schematic (right)

The optimisation objective is to minimise total system cost. This is comprised of capital costs and direct operating (fixed and variable) costs for transmission and generation. This provides a lower bound on expected actual future outlays, and does not directly consider the dynamics of the electricity market. For this reason, when it has been used with industry our model's solutions are tested using market simulations in order to evaluate generator financial viability, market spot prices and so on. Capital costs are borne in full in the year that the relevant state transition completes. Operating costs (variable and fixed) accumulate annually. For periods beyond end of planning horizon, the mathematical objective function charges an additional cost relating to ongoing costs for the generation plant inventory: this is done in order to help avoid unrealistic system run-down towards the end of the planning horizon. All costs are determined prior to the model run, and incorporate scenario-based assumptions around technology, carbon and fuel cost components over time. A discounting factor is used to discount future costs (usually $8 \%$ per annum).

Transmission and generation capacity choices are discrete and can be thought of as "projects" that the model can decide to execute. We refer to discrete options as states, and between years assets make transitions between states according to specified permissible paths, e.g., refer Figure 2. The time taken to transition between states is a parameter that can be specified on a state specific basis. That is, we can specify that a certain upgrade takes four years, for example. This will mean that a preceding transition for the asset concerned cannot occur more recently than four years prior to the transition in question. We assume that an asset can generate power 
at a state's normal capacity level during a transition from a state.

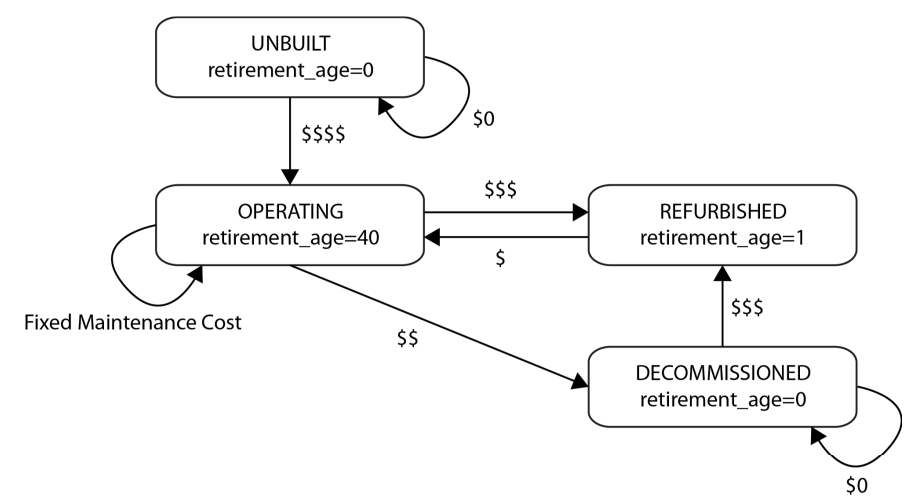

Figure 2. An example of state transitions for an electricity generation site

Data on existing power generation plant was obtained from AEMO, Geoscience Australia and the Energy Supply Association of Australia. The data was processed to create a set of 390 generation sites, including new generators to be commissioned before 2016. We then position new facilities not representing known generation projects near a zone "hub" or in a polygon for renewable resources defined for the AEMO 100\% renewables study (Australian Energy Market Operator, 2013). Although the model can represent junctions and network detail, in our current datasets all generation is connected to zone hubs by links with unrestricted capacity. The electricity demand in a zone is "sunk" at a node located at the position of the main population centre in the zone. Interconnectors (transmission lines) join the zones, and have the hubs as endpoints.

We consider centralized (CG) and decentralized (DG) generation technologies. Capacity decisions are only made with respect to CG, but DG contributes to satisfying demand. The amount of DG is dictated by solutions to the ESM model, and varies by zone and year. In all there are 36 technologies considered by our model, 24 of which are CG: several types of brown coal and black coal, open-cycle gas turbine, combined-cycle gas turbine, geothermal, wind, solar PV, solar thermal, wave energy and hydro-electric plant. Generation technologies are associated with a percentage of time a unit can be in service. For example, wind plant is assumed to be available at $90 \%$ long-run and $95 \%$ short-run (peak). This availability is one component of the conventional capacity factor view of generation capacity, where economics and energy supply availability are other factors. For wave, wind and solar, the variability of the renewable source is the biggest influence on the capacity factor.

Existing and proposed generation plant is represented by four states as per Figure 2, one of these states representing a"re-power" option for life-expired plant. Potential new generation plant has two states ("unbuilt" and "built") with capacity in the "built" state being selected from discrete graduated capacity levels or as a simple continuous variable, depending on the model variant. There are limits on generation capacity-by-technology in each zone, these coming from previously established data such as in (Australian Energy Market Operator, 2013) or from capacity "envelopes" determined by an ESM solution (our model's decisions are constrained to match the technology capacity mix determined by ESM, to a tolerance set by a user, often $-30 \% /+100 \%$ ). New generation options are developed using a complicated process driven by the need to provide the means to respect these limits. If using discrete capacity steps, most technologies end up having a site with 5-10 capacity levels in each zone. Around 600 generation sites in total are considered in a run of the model.

The transmission network topography is derived from that used by AEMO in NTNDP studies. Flow limits are directional, e.g., the transmission line between NNS and SEQ allows up to $70 \mathrm{MW}$ to flow to NNS and up to $230 \mathrm{MW}$ to flow to SEQ. Additional corridors are also considered (e.g., NSA to SWNSW). Transmission options have transitions between states of increasing capacity that are linear and irreversible. Multiple capacity-increment steps are available in each decision period: that is, a capacity jump of two or more multiples can be programmed for a year. Costs for transmission state transitions come from defined projects where known (Australian Energy Market Operator, 2012) but otherwise we have generally used an approach similar to ROAM Consulting (2011): a fixed cost of A $\$ 500,000$ per MW of augmentation is applied for most interconnectors, except for new corridors where $\mathrm{A} \$ 750,000$ is charged. We do not consider the effects that wind and ambient temperature have on transmission capacity. Connection costs for technologies in different NTNDP zones have been taken from work by Intelligent Energy Systems (2010). Line losses are modelled as a percentage loss of power flow, per length of interconnector (1\% losses per $100 \mathrm{~km})$. 


\section{ENERGY SUPPLY, DEMAND AND STORAGE}

For renewable energy supplies we utilise data that was assembled by CSIRO and ROAM Consulting for the AEMO 100\% renewables study (Australian Energy Market Operator, 2013). The land and sea area relevant to the NEM was partitioned into 43 polygons. Representative energy output traces with 30 minute time resolution for generators of different technologies were developed, for 2003-2012, in each polygon. In the experiments reported in this paper, the 2010-2011 period is used. Demand data is derived using complicated procedures and is also a time-series with 30 minute resolution that uses 2010-2011 as a basis. We do not claim any particular Probability of Exceedance (PoE) value for our demand or supply series, but the values in demand time series are inflated by $10 \%$ to qualitatively decrease the effective PoE. A system-wide reserve of $3 \mathrm{GW}$ (excess generation capacity) is applied for all years, to approximate NEM contingency planning.

Storing energy in one interval of time and releasing it in another interval of time is potentially very important for achieving reliable demand satisfaction in electricity systems, yet storage technologies are presently expensive, and if the technology uses water-pumping or geological formations, can also be restricted to certain sites (James and Hayward, 2012). Storage can operate over different timescales: short-term (order of minutes), medium-term (intra-day) or long-term (weeks or months). In non time-sequential modelling it can be difficult to address storage adequately because energy inventories cannot be tracked.

Short-term storage is used to buffer against rapid temporal variability, for example, as in (Ernst and Singh, 2008 ) to smooth the output of wind turbines. The benefits of this type of storage cannot be represented directly in the optimisation model. Storage can be used to "shift" supply within a 24 hour period: for example, to store energy during times of peak supply and discharge it either at a constant rate (for base-load), at a period of peak demand, or some combination thereof. This kind of storage can be adequately represented in our modelling approach through modifying energy supply traces, and we do this for solar thermal generation with integrated storage. Another key use of energy storage is to move energy from one relatively long interval to another, as in pumped-hydro schemes for example. In our models we do not yet address this kind of storage, although model extension options do exist.

\section{LOADBLOCKS}

For obvious computational reasons we cannot explicitly represent every hour of 35 years over 16 transmission zones and 600 generation units in a mixed-integer program and then solve it. Rather, we need to form loadblocks that are characteristic instants in time for which we must balance power flows in the network, and which represent either: (i) the average case over a subset of hours in a year; or (ii) stress cases for particular hours (future dates and times).

The purpose of the average case loadblocks is to ensure that we attain an approximate energy balance over each year and can make an estimate of annual costs and revenues in our objective function. The stress loadblocks have the role of ensuring there is adequate system generation and transmission capacity for meeting extremes in power demand and supply, within and between the regions of the overall network.

For an adequate representation of the annual energy balance and supply cost, we need to use at least eight average-case loadblocks covering the combinations of winter/summer season, day/night and weekday/weekend, in each year. In each year, a stress-case loadblock (a "metropolis peak" loadblock) of one hour duration is formed by finding the time of maximum peak in the NTNDP zones containing each of Adelaide, Melbourne, Sydney and Brisbane.

Because we are deciding on the generation mix, we do not know a-priori how much supply of each renewable technology we have in each NTNDP zone, nor where it is located (note that even with our relatively coarse consideration of the spatio-temporal variation of renewable energy supply, the time series for a generation plant in given location will be different at different locations in the NTNDP zone, because the "AEMO polygons" do not overlap the NTNDP zones). For this reason, an initial or incumbent solution specifying generator capacity, technology and location is needed for computing all stress loadblocks other than the four metropolis peak loadblocks.

Given a solution determined initially through using the eight average-case loadblocks and four metropolis peak loadblocks per year, we utilise the following heuristic approach to choose stress loadblocks.

1. A set of sets of NTNDP zones is nominated, and for each set and each hour in each year of interest, the supply from each generator (centralized) or distributed generation technology in an NTNDP zone is 
S. Dunstall et al, Accounting for renewable energy supply intermittency in energy systems modelling

evaluated and tabulated. This supply is calculated on the basis of the expected supply during a network peak period: i.e., maximum output (TSO) multiplied by the assumed availability at peak.

2. For each set of sets, the total supply per renewable energy source (groups of technologies in wave, solar, onshore wind, offshore wind and geothermal) and fossil-fuel generators (as a single group), is then calculated by summation for each hour, as is the total demand over the set of NTNDP zones, and the supply deficit, which is the value of total demand minus total supply.

3. For each set of sets, at this stage we now have an array of supply measure tuples $\{$-deficit,-demand, fossil, wave, onshore_wind, of fshore_wind, solar, geothermal $\}$ associated with specific times. Non-dominated (Pareto) minima represent potential stress loadblocks. The non-dominated tuples are identified and the top $n$ in order of non-increasing deficit selected, where $n$ depends on a threshold value of deficit and some user-specified parameters.

In practice, we use two sets of NTNDP zones: (i) all NTNDP zones combined; and (ii) SEQ and SWQ. The second set is useful due to SEQ being associated with a high peak to average demand ratio, restricted transmission capacity options, and relatively low in-situ generation plant. To control model size we only use stress loadblocks for every five years in the model: this means that the model "tests" system adequacy at five year intervals, but calculates energy balance and costs on an annual basis. The size of the MIP, and therefore the time required to solve it, is sensitive to the number of loadblocks. Up to twenty loadblocks per year - eight average-case, four metropolis peak and eight stress loadblocks — has proven adequate for yielding designs for the generation and transmission system that market simulation models verify as able to fulfil demand.

\section{THE MODEL AND ITS USE IN STUDIES}

The model has constraints for capturing physics and decision-making. The principal constraints are:

- Power balance / current balance at nodes (Kirchoff current constraints)

- Generator power output limits (based on capacity states and energy supply time-series)

- Minimum and maximum energy delivery limits (including from the ESM model)

- Line losses (function of power and line length)

- Prespecified state transitions (i.e., known commissioning and retirement)

- State transition constraints

- End-of-life repower/retirement constraints for generation plant

- Mandatory Renewable Energy Target (MRET) constraints

Mixed integer programs involving capacity constraints that link the continuous variables and binary variables are hard to solve because the LP relaxation is poor. In the experiments reported here, the MIPs are solved directly using commercial solvers. Research is continuing into the use of relaxation and decomposition techniques, which relax or avoid the capacity constraints and so extend the size of problem that can be addressed.

The insights that can be obtained through use of our model are exemplified by an ongoing study involving industry stakeholders. The stakeholders have formed statements of future scenarios (to 2050) amongst which is a "reference case" scenario where the electricity demand in the NEM grows at a moderate rate and some significant steps to address peak demand are taken (e.g., shedding of air conditioner load). 600 existing and potential generation facilities (each relating to one of the $24 \mathrm{CG}$ technologies), augmentations to existing transmission interconnectors, and several new transmission interconnectors are open for consideration.

The use of two demand cases (sub-scenarios) relating to this scenario can illustrate the influence that demand can have on the generation and transmission decisions. The first case is associated with the 2012 AEMO NTNDP and the second with a 2013 AEMO revised forecast. The 2013 demand forecast is considerably lower in total energy and peak-to-average ratio. After subtracting the output from distributed generation as specified by a preceding run of the ESM model, the annual energy demand for the NEM in the second case is 210 TWh in 2030 and $219 \mathrm{TWh}$ in 2050: for the first case the corresponding values are $225 \mathrm{TWh}$ and $258 \mathrm{TWh}$. 
For a 37-year timeframe with stress loadblocks being used for every five modelled years, and using the 2013 forecast, a MIP with 2.9 million rows (constraints), 2.75 million columns (variables) and 16.2 million nonzero coefficients was formed. Using Gurobi 5.5 as the MIP solver, the MIP presolve removes around half of the rows and columns: the resulting MIP has 0.9 million variables, with just over 100,000 of these being binary variables associated with state transition constraints and capacity constraints. Solution of the model to a $4.1 \%$ optimality gap required 48 hours in elapsed time, with the MIP solver using up to 6 parallel threads on a workstation with two $2.26 \mathrm{GHz} 8$-core Intel processors running Windows 7 . A $5 \%$ gap was reached in eight hours and the initial root-node LP solve required 3400 seconds to complete. This model performance is typical of the runs that we have carried out, and clearly indicates a need to investigate faster approaches.

In the higher demand case, six interconnectors are augmented by 2040: ADE-CVIC (500 MW), CVICSWNSW (500MW), NVIC-SWNSW (500MW), SWNSW-NCEN (500MW), NNS-SWQ (380MW to SWQ and 800MW to NNS) and SWQ-SEQ (750MW). For the lower demand case, only ADE-CVIC (1000MW) is augmented by 2050 . The relative reduction in peak demand is a key driver for this. Coal generation is largely absent by 2050, and (according to the ESM solution) gas-fired plant is preferred for supporting renewable energy supply by this date. Hydro-electric generation is constrained by water and site availability in all scenarios, and so its energy delivery remains at current levels. The adoption of solar technologies (CG and DG) is strong in the solutions, accounting for around $20 \%$ of installed capacity by 2050 , and wind generation holds a similar generation share through 2030-2050.

There is a complex trade off between transmission and generation. In some experiments, we have observed reductions in demand resulting in more transmission augmentation in NSW and Victoria, because transmission becomes cost-favourable over new generation assets. It is common for new generation to be in NSW and SW Queensland due to their centrality to the NEM, rising regional demand, and the existence of high-capacity interconnection links. The renewable energy supply does need support from fossil fuels or energy storage. In our time series based on historical patterns we see periods of very low (5\% capacity factor or less) wind supply in particular, across most of the NEM. The correlation of wind peak with demand peak is not strong, and although solar supply peak correlates with summer demand peak, there is a pinch-point in winter at dusk. Our stress loadblocks are usually found in evening winter periods except for (predominantly) Queensland daytime summer peaks where the demand is predicted to be very high.

\section{REFERENCES}

Australian Energy Market Operator (2012). 2012 National Transmission Network Development Plan.

Australian Energy Market Operator (2013). 100 Per Cent Renewables Study-Modelling Outcomes.

Binato, S., G. Couto de Oliviera, and J. Lizardo de Araújo (2001). A greedy randomized adaptive search procedure for transmission expansion planning. IEEE Transactions on Power Systems 16(2), 247-253.

Binato, S., M. V. F. Pereira, and S. Granville (2001). A new Bender's decomposition approach to solve power transmission network design problems. IEEE Transactions on Power Systems 16(2), 235-240.

Ernst, A. and G. Singh (2008). Taming wind energy with battery storage. In Annual International Conference of the German Operations Research Society, pp. 199-204.

Intelligent Energy Systems (2010). Least Cost Modelling Methodology. Prepared by IES for AEMO.

James, G. and J. Hayward (2012). AEMO 100\% Renewable Energy Study - Energy Storage. Prepared by CSIRO for AEMO.

Overbye, T., X. Cheng, and Y. Sun (2004). A comparison of the AC and DC power flow models for LMP calculations. In Proceedings of the 37th Hawaii International Conference on System Sciences, pp. 1-9.

ROAM Consulting (2011). Impact of the LRET on the costs of FCAS, NCAS and Transmission augmentation.

Romero, R., A. Monticelli, A. Garcia, and S. Haffner (2002). Test systems and mathematical models for transmission network expansion planning. IEE Proc. Generation Transmission and Distribution 159(1), $27-36$.

Short, W., P. Sullivan, T. Mai, M. Mowers, C. Uriarte, N. Blair, D. Heimiller, and A. Martinez. (2011) Regional Energy Deployment System (ReEDS). National Renewable Energy Laboratory, USA. 